\title{
A Simpler Method for Predicting Weight Loss in the First Year after Roux-en-Y Gastric Bypass
}

\author{
John P. Sczepaniak, ${ }^{1,2}$ Milton L. Owens, ${ }^{1}$ William Garner, ${ }^{2}$ \\ Farouk Dako, ${ }^{2}$ Kristin Masukawa, ${ }^{3}$ and Samuel E. Wilson ${ }^{3}$ \\ ${ }^{1}$ Coastal Center for Obesity, Orange, CA 92869, USA \\ ${ }^{2}$ John Sczepaniak Medical Enterprises, San Diego, CA 92115, USA \\ ${ }^{3}$ Long Beach Veterans Affairs Medical Center, Long Beach, CA 90822, USA \\ Correspondence should be addressed to John P. Sczepaniak, jpsczep@gmail.com
}

Received 12 July 2011; Revised 6 October 2011; Accepted 7 October 2011

Academic Editor: Pietro Forestieri

Copyright (c) 2012 John P. Sczepaniak et al. This is an open access article distributed under the Creative Commons Attribution License, which permits unrestricted use, distribution, and reproduction in any medium, provided the original work is properly cited.

\begin{abstract}
Factors postulated to predict weight loss after gastric bypass surgery, include race, age, gender, technique, height, and initial weight. This paper contained 1551 gastric bypass patients ( $85.9 \%$ female). Operations were performed by one surgeon (MLO) at community hospitals in Southern California from 1989 to 2008 with 314 being laparoscopic and 1237 open. We created the following equation: In[percent weight] $=A t^{2}-B t$, where $t$ was the time after operation (days) and $A$ and $B$ are constants. Analysis was completed on R-software. The model fits with $R^{2}$ value 0.93 and gives patients a realistic mean target weight with a confidence interval of $95 \%$ for the first year. Conclusion. We created a curve predicting weight loss after surgery as a percentage of initial weight. Initial weight was the single most important predictor of weight loss after surgery. Other recorded variables accounted for less than $1 \%$ of variability. Unknown factors account for the remaining 6-7\%.
\end{abstract}

\section{Introduction}

Bariatric surgery for weight loss in the morbidly obese is a common, major upper abdominal operations currently performed in North America [1]. Following surgery patients are often concerned to know how their weight loss compares to that of other patients. Various factors are known to influence weight loss after surgery but their relative importance is unknown [1]. From our data we developed a curve predicting weight loss after surgery as a percent of initial weight. By adding and subtracting factors known to influence weight loss and examining how they affected variability around the predicted weight loss curve we were able to assess the contribution of each factor to the predictability of the model.

\section{Patients and Methods}

The medical records of 1551 patients maintained in Microsoft Access/Excel databases (excel versions 11 and 12) who had had Roux-en-Y gastric bypass from 1989 to 2008 for morbid obesity were reviewed. Data collection included age, sex, initial weight, height, type of operation (open or laparoscopic), and subsequent weights obtained on outpatient visits. Body mass indices (BMIs) were calculated. Follow-up appointments were made for patients approximately once a month for the first three months and once every two to three months thereafter for the first year and beyond. The operations were performed by one surgeon (MLO) in nine community hospitals using standard methods previously described $[2,3]$.

To understand the relation of weight loss after gastric bypass to preoperative variables we use three alternate but relatively equivalent methods. The first method groups patients by the month after surgery and compares data in a vertical fashion; the second uses the same time groupings but looks at the linear regression between initial weight and weight for a given month; the final method creates a model based on time as a continuous variable and can be used to look at variance about the curve. 
2.1. Traditional Analysis (Method 1). In order to compare patients with different initial weights we used percent initial weight. Initial weights (IWs) were collected at or near time of surgery and all subsequent weight points were divided by IW. Only the last weight entry was taken for patients following up multiple times in a given month and used in analysis. For comparison of continuous variables such as age and height, the two outermost quartiles of patients were compared. Height, age, gender, operative approach, and race were compared at three, six, nine, and twelve months using a two-tailed Student's $t$-test. Percent IWs for Caucasian, Hispanic, and African Americans were compared using the $F$-test. Differences were considered statistically significant when the probability of the occurrence by chance was less than 0.05 .

2.2. Relation of Initial Weight to Weight after Operation (Method 2). As in traditional methods, patient data was grouped into month intervals using the latest weight data point for each patient. The patient's initial weight was plotted on the $x$-axis and weights for a particular month were plotted on the $y$-axis. Linear regression was then performed using Excel (version 12 published by the Microsoft).

2.3. Modeling Weight Trends in Time (Method 3). A plot of the data suggested that weight loss approximately followed an exponential decay, which was supported by existing literature. We took the natural log of the percent initial weight (\% IW) and fit a linear function (this is equivalent to $\%$ IW $\left.=e^{A t}\right)$. The data still had significant curvature, suggesting that a quadratic fit was more appropriate (\% IW = $\left.e^{A t^{2}-B t}\right)$. Regression was performed using the following equation: $\operatorname{In}[(\% \mathrm{IW})]=A t^{2}-B t$, where $t$ was the time after operation, measured in days and $A$ and $B$ are constants. Analysis was completed on R-software (version 2.10.1 from the R Foundation for Statistical Computing). The code for our analysis is available in Supplementary material available online at doi:10.1155/2011/195251. Data variability due to initial weight, gender, race, laparoscopic and open surgery, height, and age was examined via analysis of variance (ANOVA).

\section{Results}

Of the 1551 Roux-en-Y gastric bypass operations, 314 were performed laparoscopically and 1237 were preformed via a midline laparotomy. The open operations occurred during the early years of the study with only laparoscopic procedures done in the last eight years. There were 1332 women and 219 men. The mean age for all patients was 40.5 years (range 15.4 to 71.8 years) and the initial mean weight was $133.5 \mathrm{~kg}$ at a BMI of $48.1 \mathrm{~kg} / \mathrm{m}^{2}$ (range 79.9 to $315.1 \mathrm{~kg}$; BMI 29.9 to $98.3 \mathrm{~kg} / \mathrm{m}^{2}$ ). The initial mean weight was less for women $127.8 \mathrm{~kg}$ with BMI at $47.4 \mathrm{~kg} / \mathrm{m}^{2}$ (range 79.9 to $267.9 \mathrm{~kg}$; BMI 29.9 to $98.3 \mathrm{~kg} / \mathrm{m}^{2}$ ). Mean height was 1.64 meters for women and 1.79 meters for men.

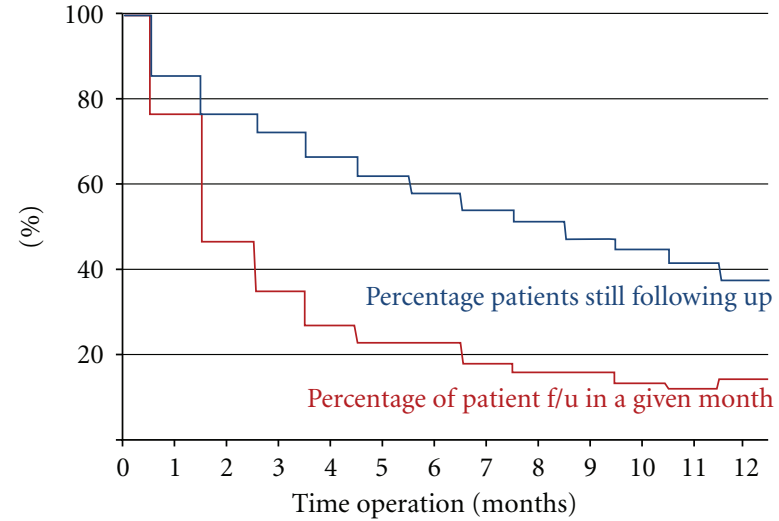

FIgure 1: Follow-up diagram. This diagram outlines the percentage of patients that were still following up in our database (blue). The diagram also notes the percentage of patients following up in any given month after surgery (red). f/u: followup.

The racial background of the patients was primarily Caucasian (940), Latino (200), African-American (137), and Asian (7), other (29), and not reported for 238 patients.

Patient followup is provided on a month by month basis in Figure 1. Figure 1 shows that $72.3 \%, 58.0 \%, 47.3 \%$, and $37.3 \%$ of patients were still following up at three, six, nine, and twelve month intervals, respectively. This is not the same as the number of patients following up in each month interval (not every patient followed up at every month). The percentage of patients following up in a given month is also provided in Figure 1.

3.1. Traditional Analysis (Method 1). The results for the traditional analysis of data are provided in Table 1. Gender was not statistically significant at any interval. The variables reaching statistical significance were age, height, race, and type of operation. Extremes in age at 12 months postoperatively had a mean difference of $6 \%(P<0.05)$. Variability in percent initial weight loss increases over time (Table 1).

3.2. Relation of Initial Weight to Weight after Operation (Method 2). The resulting linear fits are provided in Figure 2. At time zero the slope of the regression line will equal one with a perfect fit and an intercept at zero. The subsequent months have an intercept very close to zero with decreasing slopes, indicating that the greater the initial weight is the more weight that is lost and that actual weight is the same percent of initial weight at any given time after surgery. The slope represents percent initial weight. The slopes at four, eight, and twelve months are 0.81, 0.64, and 0.58, respectively.

3.3. Modeling Weight Trends in Time (Method 3). All patient data points were used up until 500 days after operation. The average number of followups per patient was $5.70 \pm 0.03$. Percentage initial weight up to 500 days is shown in Figure 3. Data show increasing variation in percent weight over time (see standard deviations in Table 1). 
TABLE 1: Method 1 results.

\begin{tabular}{|c|c|c|c|c|c|}
\hline Time after operation & 0 months & 3 months & 6 months & 9 months & 12 months \\
\hline Number of patients & 1551 & 534 & 344 & 248 & 224 \\
\hline$\%$ patients on given month & $100 \%$ & $34.4 \%$ & $22.2 \%$ & $16.0 \%$ & $14.4 \%$ \\
\hline Tallest versus shortest quartile & $\begin{array}{c}100 \% \\
\text { versus } 100 \%\end{array}$ & $\begin{array}{c}79.7 \% \\
\text { versus } 81.0 \% *\end{array}$ & $\begin{array}{c}70.7 \% \\
\text { versus } 71.7 \%\end{array}$ & $\begin{array}{c}\quad 64.4 \% \\
\text { versus } 67.5 \% *\end{array}$ & $\begin{array}{c}61.0 \% \\
\text { versus } 62.6 \% *\end{array}$ \\
\hline Youngest versus oldest quartile & $\begin{array}{c}100 \% \\
\text { versus } 100 \%\end{array}$ & $\begin{array}{c}\text { 79.4\% } \\
\text { versus } 81.0 \% *\end{array}$ & $\begin{array}{c}69.1 \% \\
\text { versus } 72.2 \% *\end{array}$ & $\begin{array}{c}\quad 63.9 \% \\
\text { versus } 66.4 \% *\end{array}$ & $\begin{array}{c}58.0 \% \\
\text { versus } 64.3 \% *\end{array}$ \\
\hline Number of males/females & $219 / 1332$ & $76 / 458$ & $57 / 287$ & $35 / 213$ & $33 / 191$ \\
\hline Males versus females \% IW & $\begin{array}{c}100 \% \\
\text { versus } 100 \%\end{array}$ & $\begin{array}{c}79.6 \% \\
\text { versus } 80.5 \%\end{array}$ & $\begin{array}{c}70.5 \% \\
\text { versus } 71.3 \%\end{array}$ & $\begin{array}{c}64.0 \% \\
\text { versus } 65.7 \%\end{array}$ & $\begin{array}{c}60.4 \% \\
\text { versus } 62.2 \%\end{array}$ \\
\hline Number of White/Hisp./Black & $940 / 200 / 137$ & $299 / 82 / 30$ & $185 / 36 / 22$ & $126 / 25 / 15$ & $117 / 20 / 14$ \\
\hline W versus $H$ versus $B$ & $100 \%$ (all) & $\begin{array}{l}82 \text { versus } 81 \\
\text { versus } 81 \%\end{array}$ & $\begin{array}{l}72 \text { versus } 72 \\
\text { versus } 75 \% *\end{array}$ & $\begin{array}{c}67 \text { versus } 66 \\
\text { versus } 71 \%\end{array}$ & $\begin{array}{l}64 \text { versus } 61 \\
\text { versus } 64 \%\end{array}$ \\
\hline Number of LAPGB/OGB & $314 / 1237$ & $135 / 399$ & $120 / 224$ & $78 / 170$ & $90 / 134$ \\
\hline LAPGB versus OGB & $\begin{array}{c}100 \% \\
\text { versus } 100 \%\end{array}$ & $\begin{array}{c}78.4 \% \\
\text { versus } 81.1 \% *\end{array}$ & $\begin{array}{c}68.6 \% \\
\text { versus } 72.6 \% *\end{array}$ & $\begin{array}{c}62.0 \% \\
\text { versus } 67.0 \% *\end{array}$ & $\begin{array}{c}59.0 \% \\
\text { versus } 63.9 \% *\end{array}$ \\
\hline Percent IW postoperation & $100 \%$ & $80.4 \%$ & $71.2 \%$ & $65.5 \%$ & $61.9 \%$ \\
\hline Standard deviation \% IW & $0 \%$ & $3.9 \%$ & $5.8 \%$ & $6.6 \%$ & $7.5 \%$ \\
\hline
\end{tabular}

* statistically significant at a 95\% confidence level

W: Whites; H: Hispanics; B: Blacks

LAPGB: laparoscopic gastric bypass

OGB: open gastric bypass

IW: initial weight.

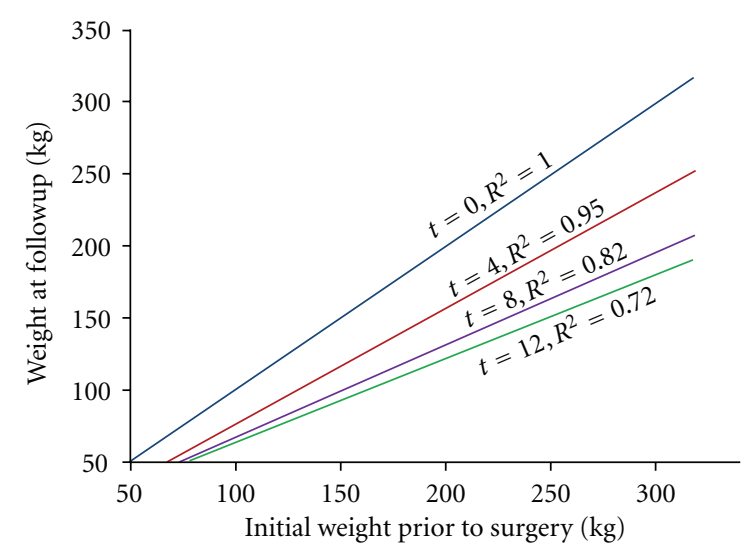

Figure 2: Linear regression of initial weight versus weight at followup. $t$ refers to the month time interval after surgery. $n$ refers to the number of patients in the linear regression model. $t=0$, $n=1551 ; t=4, n=409 ; t=8, n=245 ; t=12, n=224$.

The model predicts weight loss as a percent of initial weight and fits with an $R$ squared value of 0.93 for the first 12 months (Figure 3 ). Constants $A$ and $B$ were calculated to be $3.43 \pm 0.04 \times 10^{-6}$ and $2.57 \pm 0.01 \times 10^{-3}$, respectively. Initial weight is the most significant factor influencing weight loss and accounts for more than $93 \%$ of the variability. ANOVA results suggest race, height, gender, and type of operation (laparoscopic versus open) account for less than $1 \%$ of variability. Unknown factors account for the remaining 6$7 \%$. Variability in weight loss after one year increases over time and is probably due to influence of other factors.

\section{Discussion}

The positive results measured by reduction in various morbidities experienced by many patients electing bariatric surgery in recent years have reinforced the use of gastric bypass as a weight loss treatment. For clinical reasons the rate of weight loss following surgery is of interest. Also of interest are the relative contributions of different factors known to influence the rate of weight loss, not the least important of these being time after surgery. Recent studies investigating weight loss predictability and rate factors examined different variables individually and in combination, with results ranging from single variable positive correlations to specific profiles for postoperative success. Due to the contrasting or inconclusive findings reported in the bariatric literature, further analysis seems useful to predict weight loss following surgery.

Previous work by two groups constructed a polynomial fit for the absolute weight or BMI after surgery $[4,5]$. Using this model Dallal et al., showed that patients lost similar weight followed by an upward rebound regardless of which experienced surgeon preformed the operation.

We followed a model similar to Livingston et al. who used an exponential decay model. The formula developed by their team in 2001 was dependent on the initial fat and lean compartment mass in order to accurately predict weight loss [6]. In 2007, Chevallier et al. predicted successful procedures involving patients less than 40 years old who had an initial BMI less than $50 \mathrm{~kg} / \mathrm{m}^{2}$, combined with a willingness to change their lifestyle, and supported by an experienced bariatric surgical team [7]. Initial attempts to compare and reconcile these various bariatric surgery outcomes, however, 


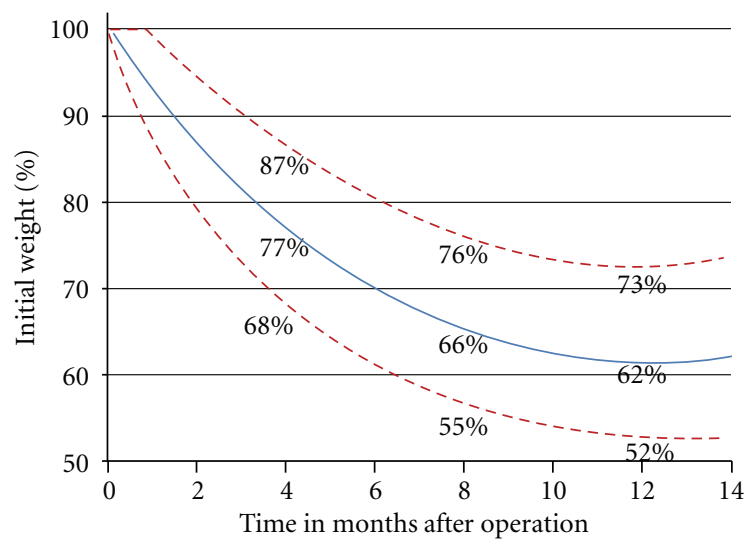

Figure 3: Regression model describing time after operation versus percent initial weight. $n=1551$. All patient data points up to 500 days after operation were used to calculate the above curve.

have been complicated by discrepancies in nomenclature, study methods, statistical detail, and definitions of weightloss success and comorbid disease resolution [8].

In our study, we observed the influence of initial weight to far exceed other factors in predicting weight loss up to one year following gastric bypass. Variability in the amount of weight lost increases over time, suggesting that continued success is dependent on additional factors.

The challenge of identifying the dominant predictors of postoperative weight loss and rate of loss stems from the range of factors influencing the patient's postoperative condition. One well-documented factor is the type of bariatric operation. A literature investigation conducted by Picot et al. in 2009 revealed gastric bypass as a more effective weight loss procedure than vertical banded gastroplasty and adjustable gastric banding, with open versus laparoscopic surgeries producing similar results in each group [9]. Furthermore, a 2010 study recommends the application of gastric bypass over adjustable gastric banding for diabetes treatment [10]. All of our patients had gastric bypass removing the type of procedure as a variable. However, our study did employ both laparoscopic and laparotomy approaches [11]. Although our laparoscopic approach produced significantly better weight loss, the addition of this and similar variables to the weight loss prediction curve increased explanation of variability only very slightly. Timing of surgery had no effect. Most of the laparoscopic procedures were done in the second half of the study period but a separate analysis of weight loss by date of surgery showed no influence due to the year in which surgery was done.

The respective roles of age and ethnicity are also areas of interest. De La Cruz-Muñoz et al. report that non-Hispanic whites and Hispanic adolescents respond positively with significant weight loss to both gastric bypass and banding surgery [12]. We did identify age and ethnicity as statistically significant variables in rate of weight loss for the first year after operation. This is similar to other studies suggesting that African American women are more resistant to weight loss than their white counter parts [13-15].
With respect to gender, Tymitz et al. report that males lose significantly more weight than females do at 6 and 12 months post operation. This correlation was weakened by the fact that the male group was significantly taller and heavier than the female group pre-op. Greater preoperative weight results in increased weight loss postoperatively as is shown by our model [16]. Our weighted model for method 3 shows an adjusted $R^{2}$ value of 0.9017 between initial weight and weight loss within the first year post-op. This correlation becomes 0.9068 when gender is added as a variable. The effect of gender on prediction of weight loss in the first year postoperation accounts for $0.51 \%$ of the variability suggesting that neither gender nor height is of much overall importance.

Other studies suggest that psychological factors also contribute to postoperative long-term success. In 2004, Tsushima et al. observed an association between the revised Minnesota Multiphasic Personality Inventory-2 and weight loss outcome one year following gastric bypass. Psychological predictors of weight loss and regain included anxiety and excessive health concerns [17]. These findings were complimented by Rutledge et al.s 2009 study, in which the assessment of mental health factors as the sum of psychiatric conditions rather than the presence of a specific condition improved the prediction of postoperative weight loss [18]. Pursuing the potential contribution of a patient's motivational characteristics, Kaiser et al. presented evidence supporting the beneficial impact of support group attendance on the percentage of excess weight loss [19]. Although our patients had psychological evaluations before and after surgery, and they attended support groups, we did not have objective data with which to measure the effect.

Alternatively, the predictive methodology itself is under scrutiny. In a 2009 study, Lee et al. demonstrated greater predictive accuracy with the decision tree model as opposed to the traditional logistical regression and discriminant analysis classification models [20]. From our perspective the approaches are complimentary rather than competitive. Regression in the period one year after gastric bypass provides a continuous model allowing patients to see the average weight decline and is more easily understood as the major variables are time and weight as related to initial weight. Decision trees are preferred when using multiple variables to predict outcomes at a single point in time.

Potential weaknesses in our model include the fact that procedures were done by one surgeon and the variability due to multiple surgeons was thereby eliminated. It follows that we were not able to assess the effect that having different surgeons might have had. We examined weight loss following gastric bypass as a single procedure and although it is tempting to think that preoperative weight is the major predictor of weight loss following other procedures we do not have data in support. Increasingly large differences in weight loss after one year make predictions based on initial weight increasingly inaccurate.

Low percent followup in our patients one year after gastric bypass may have led to a lower percent IW calculation as patients that drop out early may not lose as much weight [21]. 
In summary, we find that weight loss and thus predictability of outcome for the first postoperative year is primarily dependent on initial weight. Beyond one year, weight loss varies and is likely related to factors other than the operation such as dietary adaptation, psychiatric conditions, and physical activity. Our information may be helpful to patients and clinicians in deciding how much weight loss to expect after gastric bypass. Similar analyses for gastric banding, duodenal switch, and sleeve gastrectomy would be useful in the future.

\section{Acknowledgments}

The authors wish to thank San Diego State University and Saint George University for providing access to research materials. They are grateful for Jason Kramer's early statistical assistance and the support from UCSD and UCI Math Departments. Thanks also are due to the many others that made this work possible.

\section{References}

[1] E. H. Livingston, "The incidence of bariatric surgery has plateaued in the U.S," American Journal of Surgery, vol. 200, no. 3, pp. 378-385, 2010.

[2] M. L. Owens and J. P. Sczepaniak, "Size really does matterrole of gastrojejunostomy in postoperative weight loss," Surgery for Obesity and Related Diseases, vol. 5, no. 3, pp. 357361, 2009.

[3] B. M. Owens, M. L. Owens, and C. W. Hill, "Effect of revisional bariatric surgery on weight loss and frequency of complications," Obesity Surgery, vol. 6, no. 6, pp. 479-484, 1996.

[4] D. Jiang, K. E. Renquist, and E. E. Mason, "Weight loss curve analysis," Obesity Surgery, vol. 1, no. 4, pp. 373-380, 1991.

[5] R. M. Dallal, B. B. Quebbemann, L. H. Hunt, and L. E. Braitman, "Analysis of weight loss after bariatric surgery using mixed-effects linear modeling," Obesity Surgery, vol. 19, no. 6, pp. 732-737, 2009.

[6] E. H. Livingston, J. L. Sebastian, S. Huerta, I. Yip, and D. Heber, "Biexponential model for predicting weight loss after gastric surgery for obesity," Journal of Surgical Research, vol. 101, no. 2, pp. 216-224, 2001.

[7] J. M. Chevallier, M. Paita, M. H. Rodde-Dunet et al., "Predictive factors of outcome after gastric banding: a nationwide survey on the role of center activity and patients' behavior," Annals of Surgery, vol. 246, no. 6, pp. 1034-1039, 2007.

[8] S. A. Cunneen, "Review of meta-analytic comparisons of bariatric surgery with a focus on laparoscopic adjustable gastric banding," Surgery for Obesity and Related Diseases, vol. 4, no. 3, pp. S47-S55, 2008.

[9] J. Picot, J. Jones, J. L. Colquitt et al., "The clinical effectiveness and cost-effectiveness of bariatric (weight loss) surgery for obesity: a systematic review and economic evaluation," Health Technology Assessment, vol. 13, no. 41, pp. 215-357, 2009.

[10] E. J. Demaria, D. A. Winegar, V. W. Pate, N. E. Hutcher, J. Ponce, and W. J. Pories, "Early postoperative outcomes of metabolic surgery to treat diabetes from sites participating in the ASMBS bariatric surgery center of excellence program as reported in the bariatric outcomes longitudinal database," Annals of Surgery, vol. 252, no. 3, pp. 559-566, 2010.
[11] N. Sekhar, A. Torquati, Y. Youssef, J. K. Wright, and W. O. Richards, "A comparison of 399 open and 568 laparoscopic gastric bypasses performed during a 4-year period," Surgical Endoscopy and Other Interventional Techniques, vol. 21, no. 4, pp. 665-668, 2007.

[12] N. De La Cruz-Muñoz, S. E. Messiah, J. C. Cabrera et al., "Four-year weight outcomes of laparoscopic gastric bypass surgery and adjustable gastric banding among multiethnic adolescents," Surgery for Obesity and Related Diseases, vol. 6, no. 5, pp. 542-547, 2010.

[13] W. A. Anderson, G. W. Greene, R. A. Forse, C. M. Apovian, and N. W. Istfan, "Weight loss and health outcomes in African Americans and whites after gastric bypass surgery," Obesity, vol. 15, no. 6, pp. 1455-1463, 2007.

[14] A. K. Madan, J. D. Whitfield, J. N. Fain et al., "Are AfricanAmericans as successful as caucasians after laparoscopic gastric bypass?" Obesity Surgery, vol. 17, no. 4, pp. 460-464, 2007.

[15] H. J. Sugerman, E. J. DeMaria, J. M. Kellum, E. L. Sugerman, J. G. Meador, and L. G. Wolfe, "Effects of bariatric surgery in older patients," Annals of Surgery, vol. 240, no. 2, pp. 243-247, 2004.

[16] K. Tymitz, G. Kerlakian, A. Engel, and C. Bollmer, "Gender differences in early outcomes following hand-assisted laparoscopic Roux-en-Y gastric bypass surgery: gender differences in bariatric surgery," Obesity Surgery, vol. 17, no. 12, pp. 15881591, 2007.

[17] W. T. Tsushima, M. P. Bridenstine, and J. F. Balfour, "MMPI2 scores in the outcome prediction of gastric bypass surgery," Obesity Surgery, vol. 14, no. 4, pp. 528-532, 2004.

[18] T. Rutledge, L. M. Groesz, and M. Savu, "Psychiatric factors and weight loss patterns following gastric bypass surgery in a veteran population," Obesity Surgery, vol. 21, no. 1, pp. 29-35, 2011.

[19] K. A. Kaiser, S. F. Franks, and A. B. Smith, "Positive relationship between support group attendance and one-year postoperative weight loss in gastric banding patients," Surgery for Obesity and Related Diseases, vol. 7, no. 1, pp. 89-93, 2011.

[20] Y. C. Lee, W. J. Lee, Y. C. Lin et al., "Obesity and the decision tree: predictors of sustained weight loss after bariatric surgery," Hepato-Gastroenterology, vol. 56, no. 96, pp. 1745-1749, 2009.

[21] J. Harper, A. K. Madan, C. A. Ternovits, and D. S. Tichansky, "What happens to patients who do not follow-up after bariatric surgery?" American Surgeon, vol. 73, no. 2, pp. 181184, 2007. 


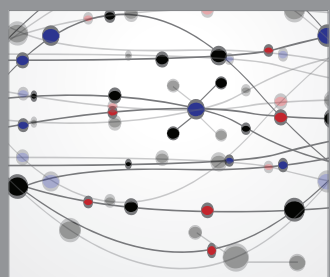

The Scientific World Journal
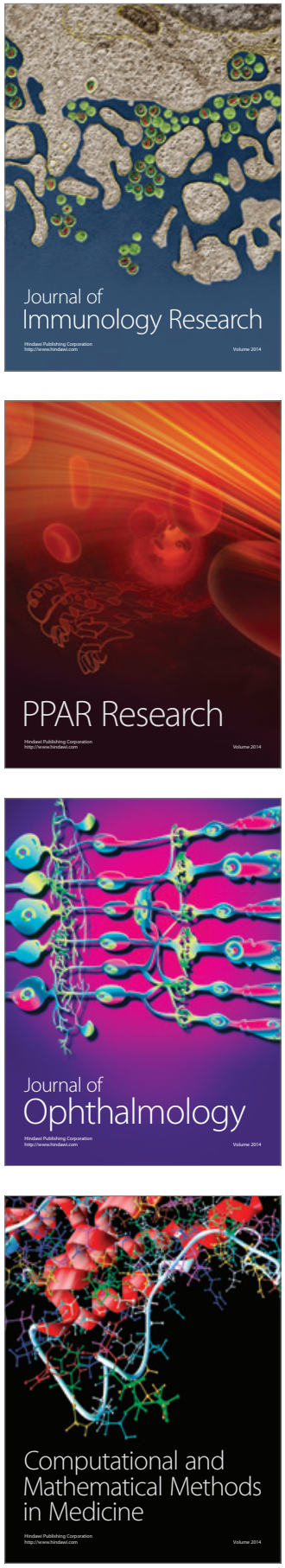

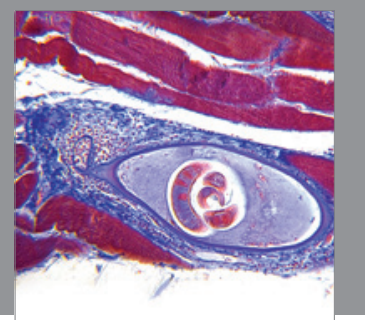

Gastroenterology

Research and Practice
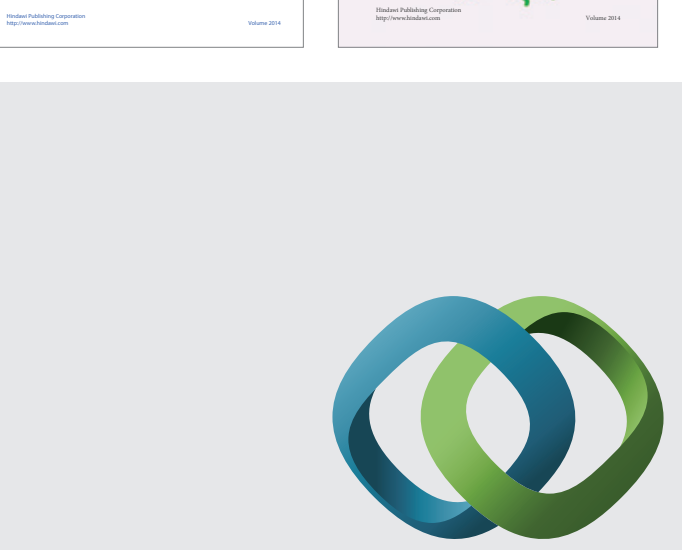

\section{Hindawi}

Submit your manuscripts at

http://www.hindawi.com
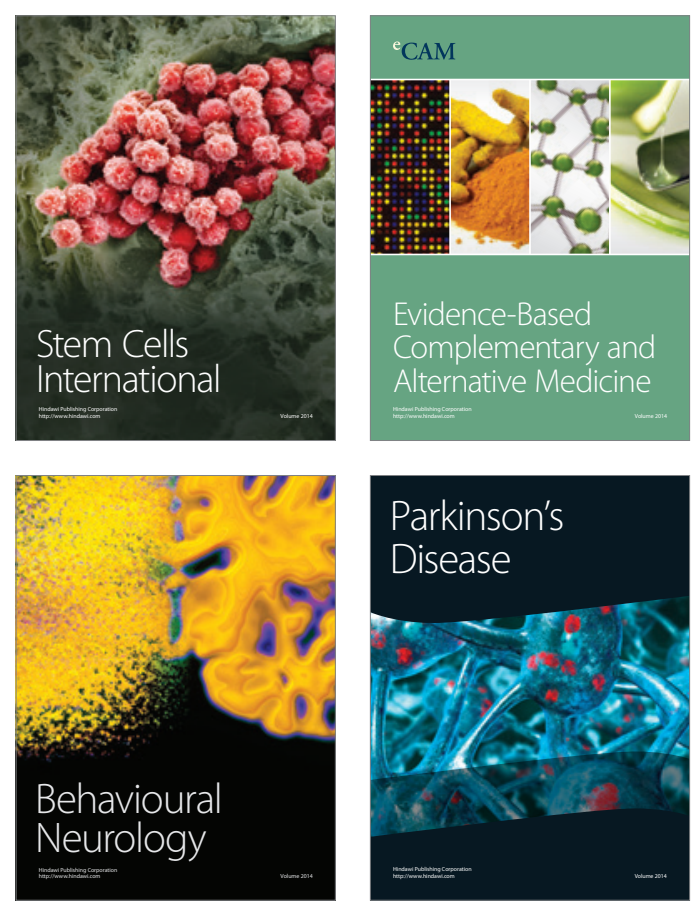

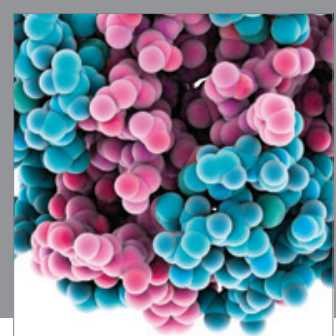

Journal of
Diabetes Research

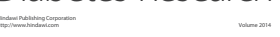

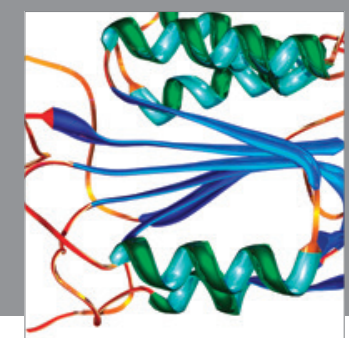

Disease Markers
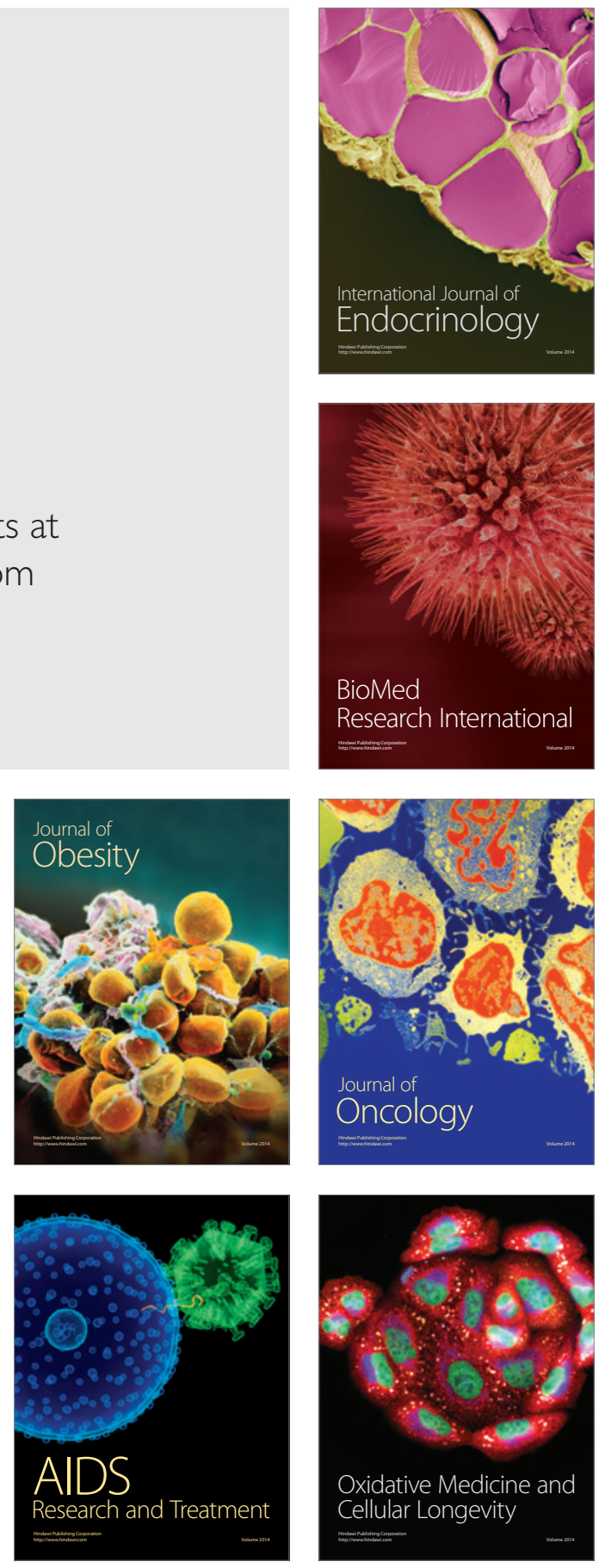